\title{
Endocytoscopy provides an in vivo virtual histo- pathological diagnosis of Whipple's disease
}

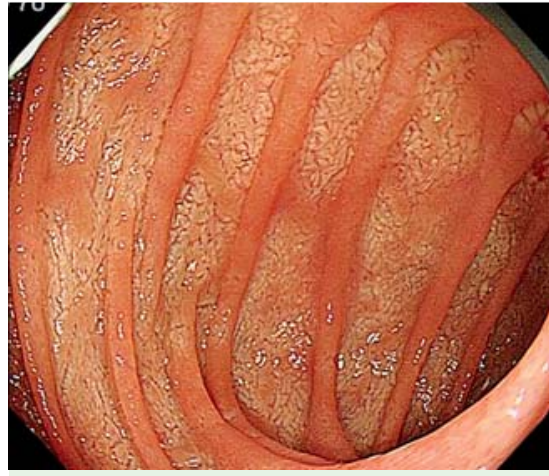

Fig. 1 Conventional high definition whitelight endoscopy of the second part of the duodenum in a 36-year-old woman who presented with fever, diarrhea, and weight loss showing yellow-white shaggy mucosa.

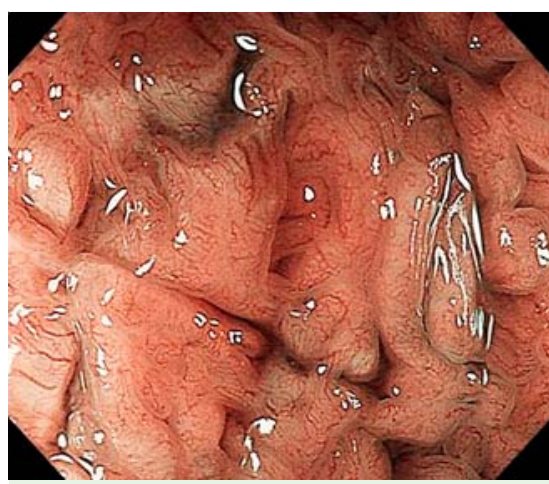

Fig. 2 Endoscopy with narrow band imaging of the second part of the duodenum showing elongated wide villi and loss of minute vessels inside each villus.
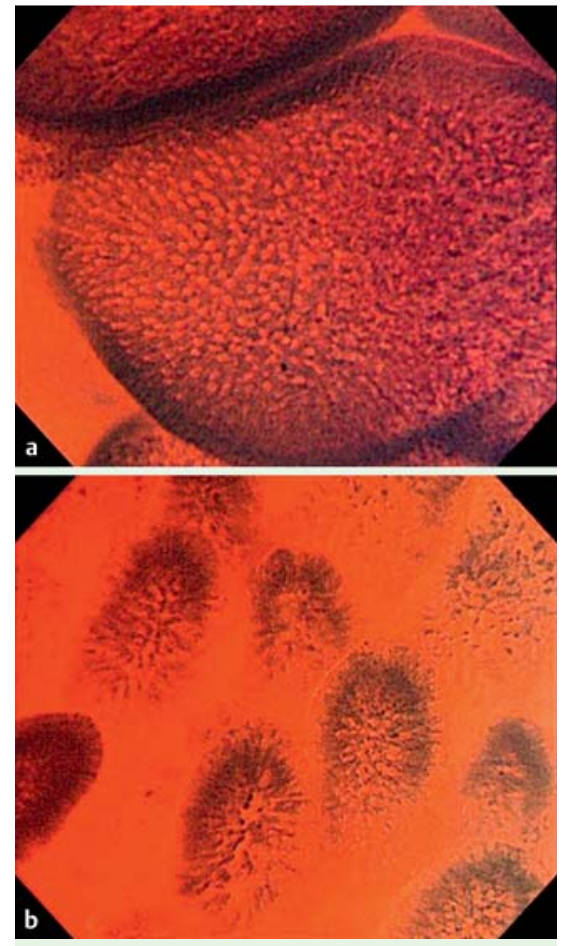

Fig. 3 Endocytoscopic view of: a Whipple's disease in the ileum showing swollen and enlarged villi; b normal ileum.

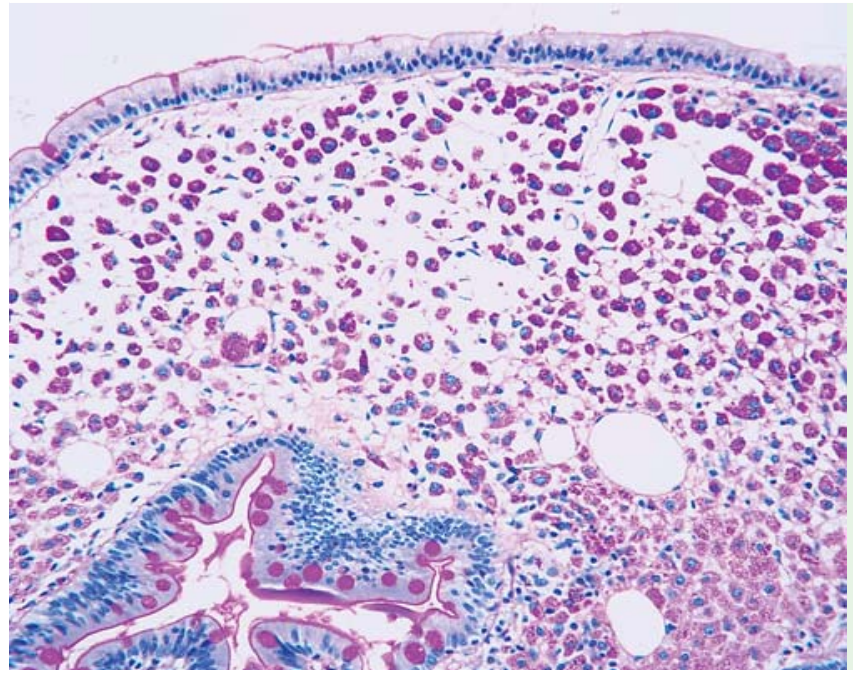

Whipple's disease is a rare multisystem malabsorption syndrome caused by Tropheryma whipplei. Whipple's disease shows pathognomonic findings on endoscopy, previously described as pale yellow shaggy mucosa [1]. Histopathology often reveals widening and flattening of the villi and infiltration of dilated lacteals. Recently, other studies have reported further endoscopic characteristics of Whipple's disease [2-4].

A 36-year-old woman presented in April 2011 with fever, diarrhea, and weight loss, so we performed endoscopy. High definition white-light endoscopy (CF-H260AZI, Olympus, Tokyo, Japan) revealed yellowwhite shaggy mucosa in the second part of duodenum ( $\mathbf{F i g . 1}$ ) and in the ileum. Optical/digital chromoendoscopy using a narrow band imaging system (Olympus) showed elongated wide villi and loss of minute vessels inside each villus ( $\bullet$ Fig.2). An endocytoscopic view at a magnification of $\times 385$ (Olympus) revealed swollen and enlarged villi $(\circlearrowleft$ Fig. 3 a) that were significantly bigger than normal ( $\bullet$ Fig. $\mathbf{3 b}$ ). In addition, there were numerous small purple spots and vacuoles inside each villus, which may correspond to foamy macrophages. The diagnosis of Whipple's disease was confirmed by periodic acid-Schiff (PAS) staining ( $\bullet$ Fig.4) and polymerase chain reaction (PCR) analysis. The patient was started on a 2-week course of ceftriaxone ( $2 \mathrm{~g} /$ day, intravenously) followed by trimethoprim-sulfamethoxazole $(4 \mathrm{~g} /$ day, orally). To date, she has gained $32 \mathrm{~kg}$ from her baseline weight.

Endocytoscopy is based on the principle of contact light microscopy and can achieve a magnification of up to $\times 385$, thereby obtaining "optical biopsies" [5]. This is the first report to characterize Whipple's disease by in vivo imaging with endocytoscopy. Whipple's disease has pathognomonic findings of flattened and widened villi, and endocytoscopy easily recognizes these mucosal alterations. Although rare, Whipple's disease should be included in the differential diagnosis of patients presenting with chronic diarrhea. The advent of endocytoscopy may aid physicians in the diagnosis of this disease without the need to take biopsies in the future.

Endoscopy_UCTN_Code_CCL_1AC_2AD

Competing interests: None 
D. Watanabe ${ }^{1}$, S. E. Kudo ${ }^{1}$, S. Hayashi' ${ }^{1}$, Y. Mori ${ }^{1}$, K. Kodama', K. Wakamura', H. Miyachi ${ }^{1}$, F. Yamamura ${ }^{1}$, K. Ohtsuka', T. Mikogami², S. Kimura ${ }^{2}$, K. Fukuchi ${ }^{3}$, N. Ikehara ${ }^{1}$

${ }^{1}$ Digestive Disease Center, Showa University Northern Yokohama Hospital, Yokohama, Japan

2 Department of Clinical Pathology, Showa University Northern Yokohama Hospital, Yokohama, Japan

${ }^{3}$ Department of Clinical Pathology, Showa University School of Medicine, Tokyo, Japan

\section{Acknowledgments}

The authors wish to thank Prof. H. Neumann for his helpful advice in the preparation of this report.

\section{References}

1 Marth T, Raoult D. Whipple's disease. Lancet 2003; 361: 239-246

2 Neufert C, Vieth $M$, Mönkemüller $K$ et al. In vivo diagnosis and characterization of Whipple's disease. Lancet Infect Dis 2011; 11: 970

3 Dolak W, Leitner J, Maresch J et al. In vivo identification by confocal laser endoscopy of foamy macrophages associated with Whipple's disease. Endoscopy 2010; 42: 310-311

4 Neumann $H$, Neufert $C$, Vieth $M$ et al. Highdefinition endoscopy with i-scan enables diagnosis of characteristic mucosal lesions in Whipple's disease. Endoscopy 2012; 44: 217-218

5 Sasajima K, Kudo S, Inoue $H$ et al. Real-time in vivo virtual histology of colorectal lesions when using the endocytoscopy system. Gastrointest Endosc 2006; 63: 1010-1017

\section{Bibliography}

Dol http://dx.doi.org/

$10.1055 / \mathrm{s}-0032-1326276$

Endoscopy 2013; 45: E143-E144

(c) Georg Thieme Verlag KG

Stuttgart · New York

ISSN 0013-726X

\section{Corresponding author}

\section{Watanabe, MD}

Digestive Disease Center

Showa University Northern Yokohama Hospital Chigasaki-chuo 35-1, Tsuzuki-ku

Yokohama 224-8503

Japan

Fax: +81-45-9497263

DW.colon@gmail.com 